\title{
PET Imaging of Perceptual Learning-Induced Changes in the Aged Rodent Cholinergic System
}

\author{
J. Miguel Cisneros-Franco 1,2*, Patrice Voss, ${ }^{1,2}$, Min Su Kang ${ }^{3,4}$, Maryse E. Thomas ${ }^{1,2}$, \\ Jonathan Côté1,2, Karen Ross' ${ }^{1}$, Pierrette Gaudreau ${ }^{5}$, David A. Rudko6, \\ Pedro Rosa-Neto ${ }^{3,4}$ and Étienne de-Villers-Sidani ${ }^{1,2 *}$ \\ ${ }^{1}$ Montreal Neurological Institute, McGill University, Montreal, QC, Canada, ${ }^{2}$ Centre for Research on Brain, Language \\ and Music, McGill University, Montreal, QC, Canada, ${ }^{3}$ Douglas Mental Health University Institute, McGill University, Montreal, \\ QC, Canada, ${ }^{4}$ Research Centre for Studies in Aging, McGill University, Montreal, QC, Canada, ${ }^{5}$ Réseau Québécois \\ de Recherche sur le Vieillissement, Université de Montréal, Montreal, QC, Canada, ${ }^{6}$ Department of Biomedical Engineering, \\ McGill University, Montreal, QC, Canada
}

OPEN ACCESS

Edited by:

Manuel S. Malmierca

University of Salamanca, Spain

Reviewed by:

Peter Brust,

Helmholtz-Zentrum

Dresden-Rossendorf (HZ), Germany

Maxime Parent,

Institut National d'Excellence en Santé et en Services Sociaux (INESSS),

Canada

*Correspondence:

J. Miguel Cisneros-Franco mike.cisneros-franco@mail.mcgill.ca

Étienne de-Villers-Sidani etienne.de-villers-sidani@mcgill.ca

Specialty section:

This article was submitted to Auditory Cognitive Neuroscience,

a section of the journal

Frontiers in Neuroscience

Received: 31 July 2019 Accepted: 20 December 2019 Published: 21 January 2020

Citation:

Cisneros-Franco JM, Voss $P$, Kang MS, Thomas ME, Côté J, Ross K, Gaudreau P, Rudko DA, Rosa-Neto $P$ and de-Villers-Sidani É (2020) PET Imaging of Perceptual Learning-Induced Changes in the Aged Rodent Cholinergic

System. Front. Neurosci. 13:1438.

doi: 10.3389/fnins.2019.01438
The cholinergic system enhances attention and gates plasticity, making it a major regulator of adult learning. With aging, however, progressive degeneration of the cholinergic system impairs both the acquisition of new skills and functional recovery following neurological injury. Although cognitive training and perceptual learning have been shown to enhance auditory cortical processing, their specific impact on the cholinergic system remains unknown. Here we used $\left[{ }^{18} \mathrm{~F}\right] \mathrm{FEOBV}$, a positron emission tomography (PET) radioligand that selectively binds to the vesicular acetylcholine transporter (VAChT), as a proxy to assess whether training on a perceptual task results in increased cholinergic neurotransmission. We show for the first time that perceptual learning is associated with region-specific changes in cholinergic neurotransmission, as detected by $\left[{ }^{18} \mathrm{~F}\right] \mathrm{FEOBV}$ PET imaging and corroborated with immunohistochemistry.

Keywords: positron emission tomography, aging, auditory cortex, operant training, somatostatin, $\left[{ }^{18} \mathrm{~F}\right] \mathrm{FEOBV}$, choline acetyltransferase (ChAT), vesicular acetylcholine transporter (VAChT)

\section{INTRODUCTION}

The rules governing experience-dependent cortical plasticity vary during the lifespan. Important structural and functional changes that occur early in life during time-limited epochs, also known as critical periods (Knudsen, 2004), perdure well into adulthood. However, the magnitude of plastic changes as well as the type of interventions that may induce plasticity, change with age. Although plasticity is still possible in the adult brain, it occurs almost exclusively in the context of learning and requires sustained attention (Bavelier et al., 2010). Enhanced cortical plasticity during adulthood can be triggered by several mechanisms including deafferentation (Chino et al., 1992; Diamond et al., 1993; Van Brussel et al., 2011), disruption in the quality or quantity of sensory input (He et al., 2006; Zhou et al., 2011), direct manipulation of neuromodulatory systems (Rokem and Silver, 2010, 2013; Kang et al., 2014; Voss et al., 2016; Chamoun et al., 2017), and perceptual learning (de Villers-Sidani et al., 2010; Mishra et al., 2014; Voss et al., 2016).

Adult neuroplasticity is partly regulated by the cholinergic system through its main neurotransmitter, acetylcholine $(\mathrm{ACh})$, which facilitates attention and learning (Hasselmo and Sarter, 2011). And like cortical plasticity, the cholinergic system is subject to significant alterations 
as it gets older. For example, aged cholinergic cells are vulnerable to degeneration (Perry, 1980; McGeer et al., 1984) leading to functional and structural damage of cortical projections (Gibson et al., 1981; Altavista et al., 1990) that have been linked to age-related cognitive and perceptual decline (Everitt and Robbins, 1997; Schliebs and Arendt, 2011). These deficits in neuromodulatory systems likely contribute to a state of dysregulated plasticity such that both diminished (Liguz-Lecznar et al., 2015) and enhanced plasticity (Cisneros-Franco et al., 2018) may occur with aging.

Recent investigations have shown that perceptual learning is a potent inducer of robust plastic changes in the aging brain, particularly in the auditory system (de Villers-Sidani et al., 2010; Mishra et al., 2014; Voss et al., 2016). However, whether these effects are mediated by neuromodulators such as the cholinergic system remains unclear. In other words, how plastic is the aging cholinergic system itself in response to perceptual learning? The purpose of the present study was to investigate how the aging rodent cholinergic system responds to perceptual learning in the auditory domain. A promising approach to achieve this goal was to use a novel positron emission tomogaphy radioligand that allows in vivo quantification of cholinergic activity throughout the brain. This radioligand, $\left[{ }^{18} \mathrm{~F}\right]$-fluoroethoxybenzovesamicol $\left(\left[{ }^{18} \mathrm{~F}\right] \mathrm{FEOBV}\right)$ is a vesamicol analog that selectively binds to the vesicular acetylcholine transporter (VAChT), a protein expressed uniquely by cholinergic neurons (Kilbourn et al., 2009). $\left[{ }^{18} \mathrm{~F}\right] \mathrm{FEOBV}$ has been used to study cholinergic activity in the context of aging and neurodegeneration both in humans (Parent et al., 2013a; Aghourian et al., 2017; Nejad-Davarani et al., 2019) and rodents (Parent et al., 2012; Cyr et al., 2014). In the present study, we scanned LOU rats, an inbred strain of Wistar origin described as a model of successful aging (Alliot et al., 2002; Boghossian et al., 2002; Garait et al., 2005; Dubeau et al., 2011). LOU rats have a longer and healthier lifespan than many other strains (Alliot et al., 2002) due to reduced incidence of metabolic, neoplastic, and cognitive disorders (Kollen et al., 2010). Here we show for the first time that perceptual learning is associated with region-specific changes in cholinergic neurotransmission detected by $\left[{ }^{18} \mathrm{~F}\right]$ FEOBV PET imaging in LOU rats, and that these changes are mirrored by anatomical correlates.

\section{MATERIALS AND METHODS}

\section{General Procedures and Study Design}

All experimental procedures used in this study were approved by the Montreal Neurological Institute Animal Care Committee and follow the guidelines of the Canadian Council on Animal Care. Aged LOU rats were housed in an environment with a 12-h light/dark cycle with unrestricted access to water. Those that underwent behavioral training were lightly food deprived. A group of rats that underwent perceptual learning training (Trained group, $n=5$, two females, 31-37 months) was compared to a control group (Untrained group, $n=10$; eight females, 30-40 months). Trained rats were scanned after an average of 12 weeks (range 9-18) of perceptual learning (60 min/day, 5 days/week), which was preceded by two behavioral shaping phases to ensure the rats could perform the auditory perceptual learning task (see "Auditory Training" section below). Training was stopped 1 day prior to the scan acquisition. Tissue collection for histological analysis was done 1-2 days after scan acquisition.

\section{Auditory Training}

Behavioral training consisted of three phases, all of which took place in an acoustically transparent operant training chamber $(60 \times 45 \times 35 \mathrm{~cm}$, length $\times$ width $\times$ height $)$ contained within a sound-attenuated chamber. During phase \#1, rats were trained to make a nose poke response to obtain a food reward. During phase \#2, rats were trained to make a nose poke only after presentation of an auditory stimulus. Phase \#3 was the actual training program, in which rats were trained to make a nose poke only for the target stimulus (a $7 \mathrm{kHz}$ pure tone) and not for a foil non-target stimulus (12 kHz pure tone). The tones were $50 \mathrm{~ms}$ in duration (5 ms cosine ramps) presented at $60 \mathrm{~dB}$ SPL, stimulus presentation was randomized, and the probability of a target stimulus presentation was $20 \%$. The protocol for the production and presentation of stimuli, data acquisition and analysis is described in Voss et al. (2016).

\section{Imaging Procedures}

$\left[{ }^{18} \mathrm{~F}\right]$ FEOBV was synthesized on scanning days at the Cyclotron Facility of the McConnell Brain Imaging Centre of the Montreal Neurological Institute (Canada). $\left.{ }^{18} \mathrm{~F}\right] \mathrm{FEOBV}$ was synthesized using a modified method (Mzengeza et al., 2007) originally described by Mulholland et al. (1993). A levo enantiomerically pure precursor (ABX advanced biochemical compounds $\mathrm{GmbH}$, Germany) was used, labeled with fluorine-18 using a SCINTOMICS (Lindach, Germany) hotbox module, resulting in $(-)-\left[{ }^{18} \mathrm{~F}\right] \mathrm{FEOBV}$, which is the only enantiomer showing affinity for VAChT (Mulholland et al., 1998). Radiochemical purity across a total of five syntheses was $97.6 \pm 1.4 \%$ (mean \pm SD). Total molar activity ranged from 404 to $1331 \mathrm{GBq} / \mu \mathrm{mol}$, whereas the mean total activity injected was $15.8 \pm 1.4 \mathrm{MBq}$.

All scans $(n=15)$ were acquired with a CTI Concorde rodent $\mathrm{R}_{4}$ microPET system (Siemens Medical Solutions). Rodents under general anesthesia (4\% isoflurane during induction, followed by $1-2 \%$ isoflurane through a nose cone) were placed on a pre-warmed stereotaxic head holder and positioned in the center of the scanner's field of view. Vital signs (temperature, heart rate, and blood pressure) were monitored throughout the procedure (BIOPAC physiological monitoring). Each PET session consisted of a brief 5 min transmission followed by a 60 min emission scan. The transmission scan was obtained using a rotating $\left[{ }^{57} \mathrm{Co}\right]$ point source. Emission scans were initiated immediately after the transmission scan with a bolus injection of $0.2 \mathrm{~mL}$ of $\left[{ }^{18} \mathrm{~F}\right] \mathrm{FEOBV}$ radiotracer in the tail vein of the rat. Transmission and emission scans were obtained using list mode acquisition. After the procedure, animals were placed under a heating light and closely monitored until full recovery.

$\mathrm{T}_{2}$-weighted structural MR images were also obtained in vivo for one rat using a 7T Bruker Pharmascan 70/16 US dedicated pre-clinical MRI system (Bruker, Billerica, MA, United States). The Pharmascan is equipped with an Avance II radiofrequency (RF) amplifier architecture. $\mathrm{T}_{2}$-weighed axial images were 
specifically acquired using a Turbo-Rapid Acquisition with Refocused Echoes (Turbo-RARE) sequence with 26 slices of $1 \mathrm{~mm}$ thickness and an in-plane resolution of $150 \mu \mathrm{m}$. Other MRI scan parameters were: $\mathrm{TE}=30 \mathrm{~ms}, \mathrm{TR}=3 \mathrm{~s}$, and a RARE factor of eight. A $640 \mathrm{~mm}$ quadrature volume transceiver RF coil was used for rat brain imaging.

\section{Imaging Processing and Analysis}

MicroPET images were histogrammed into 27 sequential time frames of increasing duration $(8 \times 30 \mathrm{~s}, 6 \times 1 \mathrm{~min}$, $5 \times 2 \mathrm{~min}, 8 \times 5 \mathrm{~min}$ ) over $60 \mathrm{~min}$. Images were reconstructed using a maximum a posteriori (MAP) algorithm, normalized and corrected for scatter, dead time and decay. The MINC software toolbox was used to perform all image analyses ${ }^{1}$. Time-averaged tissue-radioactivity images were manually coregistered to the structural $M R$ image using a seven degrees of freedom registration matrix (rigid body transformation plus one scaling constant) (Rubins et al., 2003). The microPET image outcome measure was non-displaceable binding potential $\left(\mathrm{BP}_{\mathrm{ND}}\right) . \mathrm{BP}_{\mathrm{ND}}$ was calculated using a simplified reference-tissue method (SRTM) for reversible ligands at the voxel level (Gunn et al., 1997), The cerebellar cortex served as a reference region due to its negligible amounts of cholinergic markers (Schäfer et al., 1994). The resulting images were convolved using a Gaussian kernel (FWHM $=1.2 \mathrm{~mm})$.

\section{Immunohistochemistry, Microscopy, and Data Analysis}

Tissue collection and processing were performed as previously described (Voss et al., 2016). Specific antibodies used to label brain tissue were: (1) rat anti-SOM (Millipore Sigma \#MAB354, $1: 500$ ), (2) goat anti-ChAT (Millipore Sigma \#AB144P, $1: 200$ ), (3) donkey anti-goat [conjugated to Alexa Fluor (AF647), 1 : 800, Jackson ImmunoResearch, West Grove, PA, United States], and (4) donkey anti-rat (AF488, $1: 800$, Jackson).

A Zeiss LSM 510 Meta confocal microscope equipped with filter for green Cy2/AF488, red CY3, and infrared CY5/AF647 was used to assess fluorescence in the immunostained sections. To locate the primary auditory cortex (A1) we used the stereotaxic coordinates (Paxinos and Watson, 2013): interaural between 5.76 and $2.16 \mathrm{~mm}$ and Bregma between -3.24 and $-6.84 \mathrm{~mm}$. To quantify the positive cells, 21 digital images of A1 cortical sections were taken with a $40 \times$ objective (Zeiss LSM 510) at random locations within each $\mathrm{A} 1$ of both hemispheres for each animal. All quantifications were assessed in 400-500 $\mu \mathrm{m}$ wide A1 sectors (the approximate width of A1 on coronal sections) per hemisphere extending from layer 1 to the underlying white matter. Confocal images were thresholded and adjusted for brightness to maximize the dynamic range of each channel using Image ${ }^{2}$ and Adobe Photoshop CS5 (Adobe, San Jose, CA, United States).

We determined the number of immunolabeled cells in each section of A1 using the optical dissector method (Stereo Investigator software, MBF Bioscience, Williston, VT,

${ }^{1}$ http://www.bic.mni.mcgill.ca/ServicesSoftware/MINC
${ }^{2}$ http://rsb.info.nih.gov/ij/
United States) to avoid biased sampling. These counts were then pooled and adjusted to reflect what would have been counted in the whole $40 \times$ field. Data were then recorded as an averaged value per high power field (hpf) for each animal and group. All cells displaying labeling above background levels were counted, regardless of their staining intensity. Data from both hemispheres were pooled. An observer blind to the group membership of the animals performed all cell counts.

\section{Data and Statistical Analysis}

Regional brain differences in $\left[{ }^{18} \mathrm{~F}\right] \mathrm{FEOBV} \mathrm{BP}_{\mathrm{ND}}$ were assessed using a regions of interest (ROI)-based analysis, via a repeated measures model with group (trained, $n=5$; untrained, $n=10$ ) as the predictor variable, and ROI and hemisphere (left or right) as within-subject variables. The effects of group and ROI were calculated with a repeated-measures ANOVA. Overall and layerbased group differences in ChAT- and SST-positive cell counts were assessed in a subset of rats (trained, $n=3$; untrained, $n=6$ ) using a two-way repeated-measures ANOVA with group and layer as factors. Group $\times$ ROI/layer multiple comparisons were corrected with Tukey-Kramer's test.

\section{RESULTS}

Through training, rats learned to perform a nose poke only for the target stimulus ( $7 \mathrm{kHz}$ pure tone) and not for a foil nontarget stimulus (12 kHz pure tone; Figure 1A). The behavioral results illustrated in Figures $\mathbf{1 B}, \mathbf{C}$ show that both poke rate (the number of pokes per trial) and false-alarm rate (spontaneous pokes not preceded by any auditory stimuli) decreased over time, suggesting adequate procedural learning; i.e., rats successfully learned the association between training stimuli and rewards. All trained rats showed learning effects and generally improved steadily over the training period (Figures 1D-F). Prior to undergoing a PET scan, all trained animals had reached a criterion of d-prime $>1$, suggesting adequate perceptual learning; i.e., successful discrimination between target and nontarget stimuli.

In order to quantify $\left[{ }^{18} \mathrm{~F}\right] \mathrm{FEOBV}$ distribution in the rat brain, the following ROI were segmented using high resolution MR images of (see Figure 2): (1) frontal cortex; (2) temporoparietal cortex; (3) ventro-orbital cortex; (4) visual cortex; (5) auditory cortex; (6) nucleus basalis (7) striatum; (8) hippocampus, and (9) thalamus. The regional non-displaceable binding potential $\left(\mathrm{BP}_{\mathrm{ND}}\right)$ in all rats/scans was computed for each ROI in each hemisphere (resulting in 18 ROIs per rat/scan). An ROI of the cerebellum was also traced manually and used as the reference region for $\mathrm{BP}_{\mathrm{ND}}$, because of its negligible amounts of cholinergic markers (Schäfer et al., 1994). In agreement with previous reports, the regions with the highest tracer binding were the striatum and nucleus basalis (Parent et al., 2012; Cyr et al., 2014).

$\left[{ }^{18} \mathrm{~F}\right] \mathrm{FEOBV}$ binding potentials were quantified for each ROI and compared between groups. For group comparisons, the average $\mathrm{BP}_{\mathrm{ND}}$ in each $\mathrm{ROI}$ in each hemisphere was considered as a data point (two data points per rat/scan per ROI). A repeated measures model was used, where the repeated 

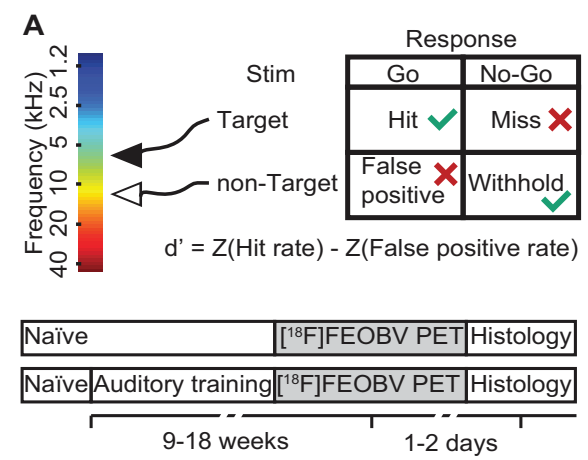

D

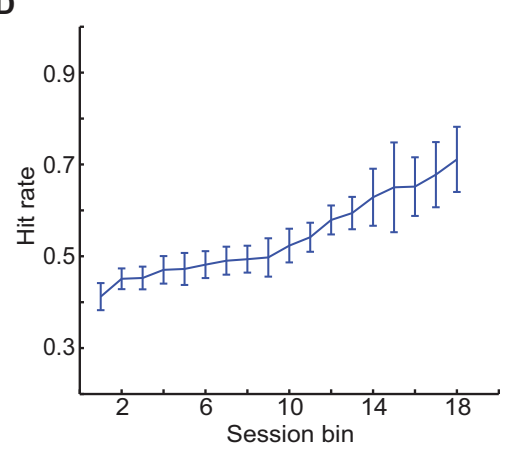

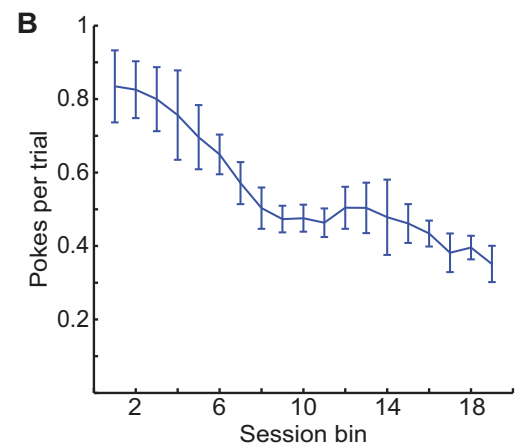

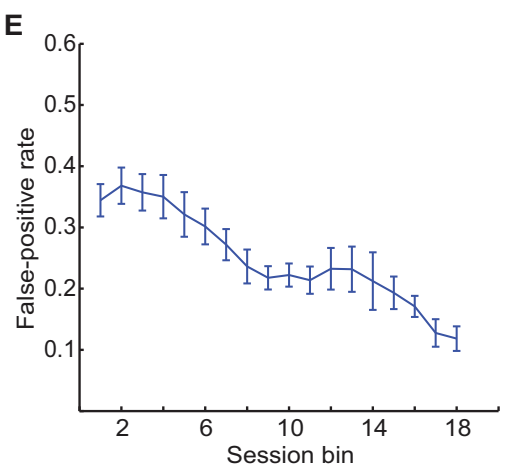

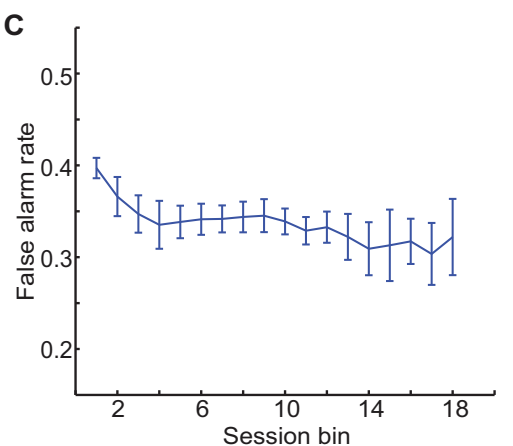

$\mathbf{F}$

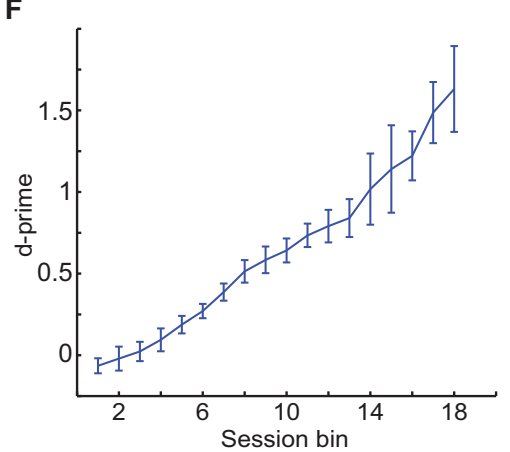

FIGURE 1 | Behavioral training results on an auditory discrimination training task. (A) Experimental paradigm. Both (B) poke rate and (C) false alarm rate decreased with training, suggesting that rats successfully learned the association between auditory stimuli and food reward. Group behavioral performance, as measured by (D) hit rate, (E) false-positive rate, and (F) d-prime. Values are mean $\pm \mathrm{SEM}, n=5$ rats.

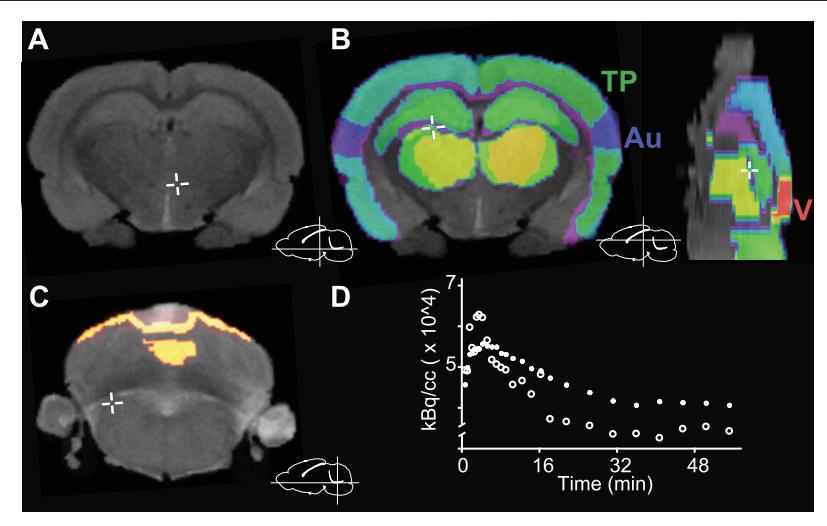

FIGURE 2 | Image processing and anatomical structure segmentation. (A) 7T in vivo MR images were obtained and (B) used for manual segmentation into nine regions of interest (ROIs). (C) The cerebellum was used as reference region for data analysis. (D) Representative time activity curve of the reference region (cerebellum, open circles) and the rest of the brain (all ROls except cerebellum, filled circles). Au, auditory cortex; TP, temporoparietal cortex; $\mathrm{V}$, visual cortex. Insets (A-C) depict the position of the cursor (cross) for each panel.

measures corresponded to the 18 individual ROIs per rat/scan, group was the predictor variable, and the within-subjects variables were ROI and hemisphere (left or right). There were no significant differences in overall $\left[{ }^{18} \mathrm{~F}\right] \mathrm{FEOBV} \mathrm{BP}_{\mathrm{ND}}$ as a function of group or hemisphere (both $p \geq 0.25$, simple main effects test). Further group $\times$ ROI analysis revealed a significant increase in $\left[{ }^{18} \mathrm{~F}\right] \mathrm{FEOBV} \mathrm{BP}_{\mathrm{ND}}$ exclusively in the auditory cortex of the trained group, relative to controls $(p=0.038$; all other ROIs, $p \geq 0.17$, with Tukey-Kramer test for multiple comparisons; Figure 3).

To obtain histological confirmation of the effect of training on the cholinergic system, we compared the density of choline acetyl-transferase (ChAT) staining in A1 sections obtained from both untrained $(n=6)$ and trained rats $(n=3$; Figure $4 \mathrm{~A}$, left). As reported earlier (Voss et al., 2016), ChAT density was significantly higher in trained rats $(863.95 \pm 245.87$ optical density arbitrary units, a.u.) compared to untrained ones (772.67 \pm 237.91 a.u., $p=0.041$, two-way ANOVA; Figure $4 \mathbf{B}$ ), consistent with both our above results and previous imaging findings (Parent et al., 2013b).

To determine whether increased density of ChAT staining might be related to increased cholinergic innervation onto A1, we performed quantitative analysis of the density of ChATpositive $(\mathrm{ChAT}+)$ cells across layers. However, we did not find any significant differences in the average number of ChAT immunoreactive cells per A1 high power field (hpf) between trained and untrained rats (Figure 4C). Somatostatin-positive $(\mathrm{SST}+)$ cells, the second largest interneuron subpopulation, are the primary targets of cholinergic afferents to the cortex, and contribute to the neuromodulation of sensory processing during both passive sound exposure (Chen et al., 2015) and operant training (Fu et al., 2015). For this reason, we also quantified the density of SST+ cells per hpf (Figure 4A, right). In line 


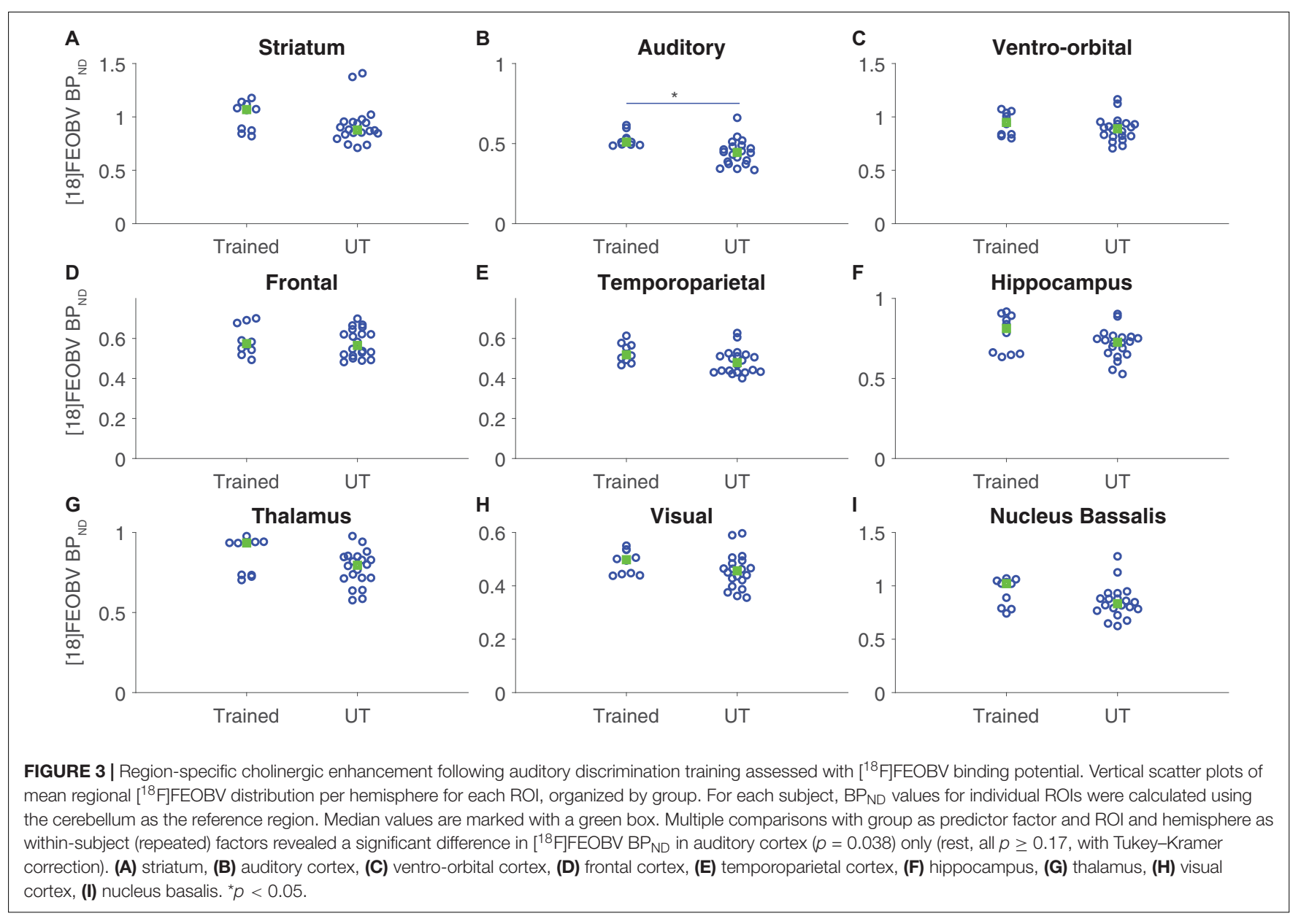

with our results on ChAT+ cells, the control and experimental groups did not differ in overall nor layer-specific SST+ cell density (Figure 4D). Taken together, these results suggest that training-related changes in $\left[{ }^{18} \mathrm{~F}\right] \mathrm{FEOBV}$ binding detected by PET imaging were associated with an overall increase in cholinergic neurotransmission, without any layer-specific changes.

\section{DISCUSSION}

The purpose of the present study was to investigate how the aging rodent cholinergic system responds to auditory perceptual learning using a novel PET radioligand that selectively binds to VAChT. We showed that auditory perceptual learning increases cholinergic neurotransmission within the aged auditory cortex as assessed via both $\left[{ }^{18} \mathrm{~F}\right] \mathrm{FEOBV}$ PET imaging and immunochemistry. Our findings suggest that auditory perceptual learning significantly increased cholinergic neurotransmission in auditory cortex only, and not in any of the other ROIs. To our knowledge, this is the first use of $\left[{ }^{18} \mathrm{~F}\right] \mathrm{FEOBV}$ in rodents to demonstrate how perceptual learning can modulate the expression of $\mathrm{ACh}$ in a region-specific manner.

This increase in ACh binding following training sharply contrasts with the more typical age-related decline in binding observed with aging. Indeed, aging is associated with the degeneration of cholinergic cells (Perry, 1980; McGeer et al., 1984) and their projections (Gibson et al., 1981; Altavista et al., 1990). These age-related changes in cholinergic neurotransmission likely contribute to the attentional and cognitive deficits observed during aging (Everitt and Robbins, 1997; Schliebs and Arendt, 2011). This hypothesis is supported by converging evidence from various lines of research on the long-term effects of manipulating cholinergic neurotransmission in learning and attention. First, the activation of the cholinergic system during perceptual training leads to a long-lasting shaping of cortical circuits that forms the basis of learning (Rokem and Silver, 2013). Indeed, neurochemically boosting cholinergic transmission (Greuel et al., 1988; Furey et al., 2008; Rokem and Silver, 2010) and stimulating the basal forebrain (Greuel et al., 1988; Furey et al., 2008; Rokem and Silver, 2010) have both been shown to have a significant effect on both learning and the cortical processing of stimuli. Second, perceptual learning can itself produce long-lasting changes in perceptual abilities (Goldstone, 1998; Ohl and Scheich, 2005). Third, selective lesions of cholinergic neurons in the rat pedunculopontine tegmental nucleus resulted in attentional deficits that were proportional to the extent of neuronal loss (Cyr et al., 2015). Consequently, in general, 


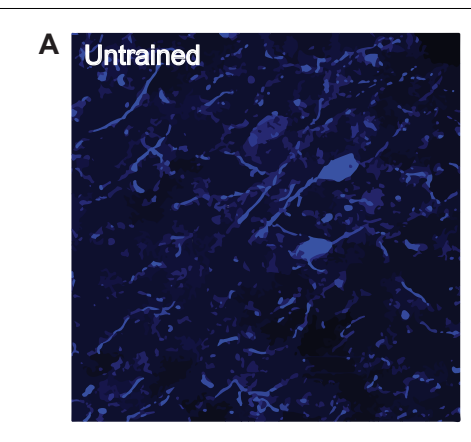

B

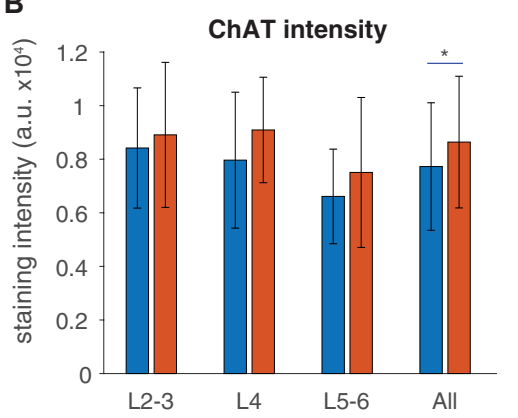

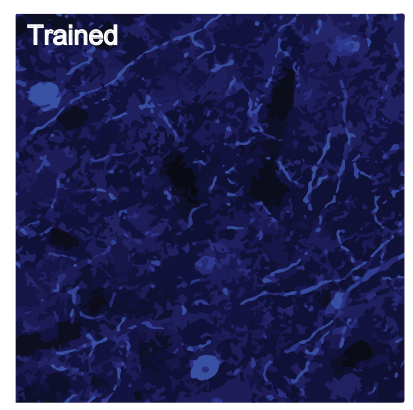
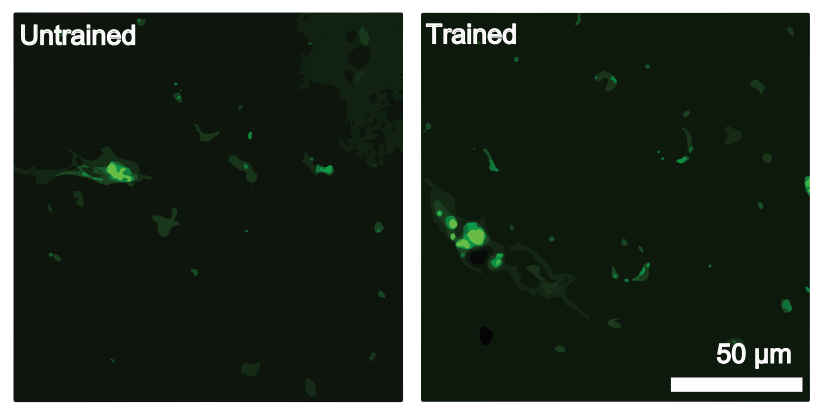

Untrained Trained C

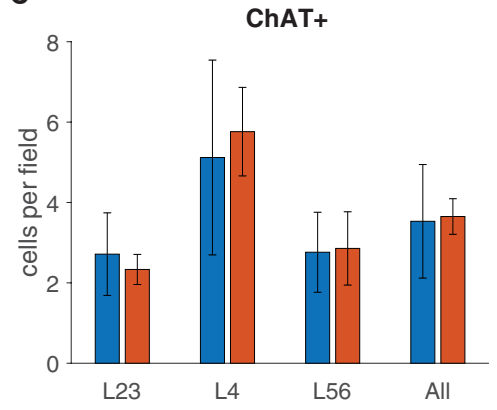

D

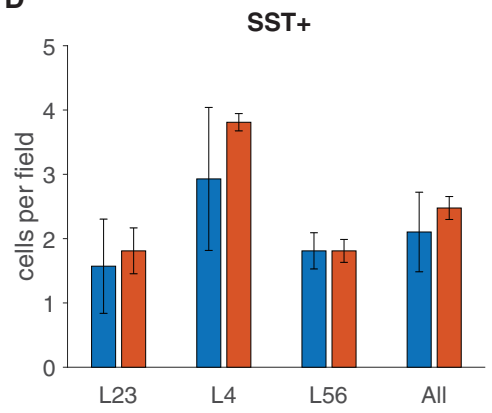

FIGURE 4 | Increased intensity of ChAT staining in A1 following auditory discrimination training. (A) High-power microphotographs of A1 sections stained for choline acetyl-transferase (ChAT, left) and somatostatin (SST, right). (B) Increased overall ChAT staining intensity in the trained group (effects of group: $F(1,21)=4.72$, $p=0.041$; layer: $F(2,21)=3.44, p=0.051$; interaction, $F(2,21)=0.31, p=0.73$; two-way ANOVA). Group, number of subjects, high-power fields (hpf), and individual data points: Untrained, 6, 126, 446; Trained, 3, 63, 230. (C) ChAT+ cell count: two-way rmANOVA with group and layer as factors. Effects of layer: $F(2,14)=17.58, p=0.0001$; group: $F(1,7)=0.01, p=0.9$; interaction: $F(2,14)=0.44, p=0.64$. (D) SST+ cell count: two-way rmANOVA with group and layer as factors. Effects of layer: $F(2,14)=22.03, p<0.001$; group: $F(1,7)=0.81, p=0.39$; interaction: $F(2,14)=1.3, p=0.3$. Group, number of subjects, hpf for SST/ChAT+ cell counts: Untrained, 6, 126; Trained, 3, 63. Values shown are mean \pm standard deviation. * $p<0.05$.

ACh is believed to mediate voluntary attention, a major requisite for adult learning (Hasselmo and Bower, 1993; Sarter et al., 2005).

Recent studies have shown that, on shorter time-scales, fast central cholinergic responses observed in sensory cortex during learning might in fact convey reinforcement- and reward-elicited signals that enable the brain to associate prior events with behavioral outcome, thereby promoting local cortical plasticity and learning (Hangya et al., 2015; Liu et al., 2015). Of note, such precisely timed cholinergic signaling onto sensory cortex appears to be essential for sustained performance even after the completion of training (Kuchibhotla et al., 2017). Given the time scale of the present study, both types of cholinergic influence - on attention and reinforcement - are likely to have played a role in promoting plasticity and learning. However, future studies are warranted in order to disentangle the relative attentional and reinforcement effects of cholinergic input during perceptual learning.

As highlighted above, we found elevated cholinergic binding in the auditory cortex following perceptual learning. Although peak $\left[{ }^{18} \mathrm{~F}\right] \mathrm{FEOBV}$ levels are observed within the first $5 \mathrm{~min}$ post-injection in all brain regions, previous experiments have shown that relevant mapping of VAChT concentrations can be reliably obtained within a $60 \mathrm{~min}$ acquisition (Mulholland et al., 1998; Kilbourn et al., 2009).
Furthermore, $\left[{ }^{18} \mathrm{~F}\right] \mathrm{FEOBV}$ binding distribution in the brain corresponds to the known anatomical distribution of cholinergic terminals (Kilbourn et al., 2009; Parent et al., 2012, 2013b). Moreover, $\left[{ }^{18} \mathrm{~F}\right] \mathrm{FEOBV}$ PET can detect cholinergic depletion, whether it be following lesions of the nucleus basalis (Cyr et al., 2014) or in association with aging (Parent et al., 2012), deficits that were also confirmed with ChAT immunostaining (Parent et al., 2013b). Consequently, current evidence suggests that $\left[{ }^{18} \mathrm{~F}\right] \mathrm{FEOBV}$ can reliably be used to assess both the integrity and the learning-induced changes of the ACh system.

Our anatomical findings of an overall ChAT staining intensity increase in the trained animals are consistent with our $\left[{ }^{18} \mathrm{~F}\right]$ FEOBV PET findings, and suggest that training-induced plasticity in the cholinergic system was indeed characterized by an overall increase in A1 ACh neurotransmission. We did not find any layer-specific group differences in ChAT staining intensity, albeit a non-statistically significant increase was observed for all layers (see Figure 4). Although the present findings do not allow us to conclude on the matter, recent evidence suggests that the cholinergic control of cortical synaptic plasticity may be layer-specific (Bloem et al., 2014), whereby cholinergic stimulation produces opposite plasticity modulation effects on the superficial and deep cortical layers (Verhoog et al., 2016; Obermayer et al., 2017). 
One of the more robust explanations of the role of $\mathrm{ACh}$ in perceptual learning posits that learning-related plasticity is achieved by a cholinergic-mediated shift in the cortical excitatory/inhibitory balance such that the input of afferent excitatory projections is favored over that from lateral intracortical connections (Hasselmo and Bower, 1993). A wealth of studies support this theory, and have specifically shown that cortical interneuron activity is modulated by cholinergic afferents (Kawaguchi, 1997; Fanselow et al., 2008; Kuchibhotla et al., 2017). In contrast to our ChAT staining intensity findings and our previous report of increased SST+ cell counts with auditory training (Voss et al., 2016), we found no significant changes in SST + cell density in the present study. At least three factors might have contributed to this discrepancy. The first two factors relate to the strain and age of experimental subjects (Brown Norway rats, all $\leq 30$ months in our previous study vs. LOU rats, all $\geq 30$ months in the present investigation). Finally, although similar learning-related increases in SST + cell density have been reported in young adult barrel cortex (Cybulska-Klosowicz et al., 2013), it is not clear whether perceptual learning is associated with neurogenesis in adult neocortex (Greene et al., 2019). The latter observation would also explain, at least in part, the unchanged ChAT + cell counts in our trained group. In light of these mixed results, further studies are needed to clarify whether there is a causal link between cholinergic neurotransmission and SST + cell density. Future studies may also explore the possible differential recruitment of cortical interneurons in a layer-specific manner by cholinergic inputs and their contribution to auditory perceptual learning.

Even though the present study is the first use of $\left[{ }^{18} \mathrm{~F}\right] \mathrm{FEOBV}$ in rodents to demonstrate how perceptual learning can modulate the expression of $\mathrm{ACh}$, some limitations are worthy of mention. The primary limitation is the cross-sectional design. The rationale behind this design choice stems from the fact that this study was dependent on leftover $\left[{ }^{18} \mathrm{~F}\right] \mathrm{FEOBV}$ synthesized for ongoing human studies, making it difficult to predict when the next scans would occur. And although we were still able to identify perceptual learning-related increases in VAChT expression, a longitudinal design would yield a more accurate picture of $\mathrm{ACh}$ system changes as a function of learning. Furthermore, a longitudinal design would allow for a direct correlation between ACh binding and learning efficiency. Future studies may also want to compare $\left[{ }^{18} \mathrm{~F}\right] \mathrm{FEOBV}$ binding prior to and immediately after training to investigate the short-term changes produced in the cholinergic system by perceptual learning.

\section{REFERENCES}

Aghourian, M., Legault-Denis, C., Soucy, J.-P., Rosa-Neto, P., Gauthier, S., Kostikov, A., et al. (2017). Quantification of brain cholinergic denervation in Alzheimer's disease using PET imaging with [F]-FEOBV. Mol. Psychiatry 22, 1531-1538. doi: 10.1038/mp.2017.183

Alliot, J., Boghossian, S., Jourdan, D., Veyrat-Durebex, C., Pickering, G., MeynialDenis, D., et al. (2002). The LOU/c/jall rat as an animal model of healthy aging? J. Gerontol. A Biol. Sci. Med. Sci. 57, B312-B320.

Altavista, M. C., Rossi, P., Bentivoglio, A. R., Crociani, P., and Albanese, A. (1990). Aging is associated with a diffuse impairment of forebrain cholinergic neurons. Brain Res. 508, 51-59. doi: 10.1016/0006-8993(90)91116-x

\section{CONCLUSION}

In conclusion, we believe our study provides proof of principle data that $\mathrm{PET}$ combined with the $\left[{ }^{18} \mathrm{~F}\right] \mathrm{FEOBV}$ radioligand can be used to assess changes in cholinergic neurotransmission induced by neuroplastic learning processes.

\section{DATA AVAILABILITY STATEMENT}

The datasets generated for this study are available on request to the corresponding author. Data have been deposited in the online open access repository Mendeley Data: https://data.mendeley. com/datasets/ddm $47 \mathrm{dxms} 7 / 1$.

\section{ETHICS STATEMENT}

The animal study was reviewed and approved by the Montreal Neurological Institute Animal Care Committee.

\section{AUTHOR CONTRIBUTIONS}

JC-F and ÉD-V-S: conceptualization. MK, KR, DR, and PR-N: methodology. JC-F, MK, and MT: formal analysis. JC-F, MK, MT, JC, and KR: investigation. PG, DR, PR-N, and ÉD-V-S: resources. PV: writing - original draft. JC-F, PV, MT, and ÉD-V-S: writing review and editing. PR-N and ÉD-V-S: supervision. ÉD-V-S: funding acquisition.

\section{FUNDING}

This research was supported by the Canadian Institutes of Health Research (CIHR, Grant MOP-133426 to ÉD-V-S and a CIHR Vanier Canada Graduate Scholarship to JC-F). The Centre for Research on Brain, Language and Music is funded by Fonds de Recherche du Québec-Nature et Technologies and Fonds de Recherche du Québec-Société et Culture.

\section{ACKNOWLEDGMENTS}

We thank Lydia Ouellet for help with immunohistochemistry. We also thank Gassan Massarweh and Arturo Aliaga for excellent technical assistance with neuroimaging experiments.

Bavelier, D., Levi, D. M., Li, R. W., Dan, Y., and Hensch, T. K. (2010). Removing brakes on adult brain plasticity: from molecular to behavioral interventions. J. Neurosci. 30, 14964-14971. doi: 10.1523/JNEUROSCI.4812-10. 2010

Bloem, B., Schoppink, L., Rotaru, D. C., Faiz, A., Hendriks, P., Mansvelder, H. D., et al. (2014). Topographic mapping between basal forebrain cholinergic neurons and the medial prefrontal cortex in mice. J. Neurosci. 34, 16234-16246. doi: 10.1523/JNEUROSCI.3011-14.2014

Boghossian, S., Nzang Nguema, G., Jourdan, D., and Alliot, J. (2002). Old as mature $\mathrm{LOU} / \mathrm{c} / \mathrm{jall}$ rats enhance protein selection in response to a protein deprivation. Exp. Gerontol. 37, 1431-1440. doi: 10.1016/s0531-5565(02)00 $174-2$ 
Chamoun, M., Huppé-Gourgues, F., Legault, I., Rosa-Neto, P., Dumbrava, D., Faubert, J., et al. (2017). Cholinergic potentiation improves perceptualcognitive training of healthy young adults in three dimensional multiple object tracking. Front. Hum. Neurosci. 11:128. doi: 10.3389/fnhum.2017.00128

Chen, I.-W., Helmchen, F., and Lütcke, H. (2015). Specific early and late oddballevoked responses in excitatory and inhibitory neurons of mouse auditory cortex. J. Neurosci. 35, 12560-12573. doi: 10.1523/JNEUROSCI.2240-15.2015

Chino, Y. M., Kaas, J. H., Smith, E. L. III, Langston, A. L., and Cheng, H. (1992). Rapid reorganization of cortical maps in adult cats following restricted deafferentation in retina. Vision Res. 32, 789-796. doi: 10.1016/0042-6989(92) 90021-a

Cisneros-Franco, J. M., Ouellet, L., Kamal, B., and de Villers-Sidani, E. (2018). A brain without brakes: reduced inhibition is associated with enhanced but dysregulated plasticity in the aged rat auditory cortex. eNeuro 5:ENEURO.005118.2018. doi: 10.1523/ENEURO.0051-18.2018

Cybulska-Klosowicz, A., Posluszny, A., Nowak, K., Siucinska, E., Kossut, M., and Liguz-Lecznar, M. (2013). Interneurons containing somatostatin are affected by learning-induced cortical plasticity. Neuroscience 254, 18-25. doi: 10.1016/ j.neuroscience.2013.09.020

Cyr, M., Parent, M. J., Mechawar, N., Rosa-Neto, P., Soucy, J.-P., Aliaga, A., et al. (2014). PET imaging with [18F]fluoroethoxybenzovesamicol ([18F]FEOBV) following selective lesion of cholinergic pedunculopontine tegmental neurons in rat. Nucl. Med. Biol. 41, 96-101. doi: 10.1016/j.nucmedbio.2013.10.004

Cyr, M., Parent, M. J., Mechawar, N., Rosa-Neto, P., Soucy, J.-P., Clark, S. D., et al. (2015). Deficit in sustained attention following selective cholinergic lesion of the pedunculopontine tegmental nucleus in rat, as measured with both post-mortem immunocytochemistry and in vivo PET imaging with [18F] fluoroethoxybenzovesamicol. Behav. Brain Res. 278, 107-114. doi: 10. 1016/j.bbr.2014.09.021

de Villers-Sidani, E., Alzghoul, L., Zhou, X., Simpson, K. L., Lin, R. C. S., and Merzenich, M. M. (2010). Recovery of functional and structural age-related changes in the rat primary auditory cortex with operant training. Proc. Natl. Acad. Sci. U.S.A. 107, 13900-13905. doi: 10.1073/pnas.1007885107

Diamond, M. E., Armstrong-James, M., and Ebner, F. F. (1993). Experiencedependent plasticity in adult rat barrel cortex. Proc. Natl. Acad. Sci. U.S.A. 90, 2082-2086.

Dubeau, S., Ferland, G., Gaudreau, P., Beaumont, E., and Lesage, F. (2011). Cerebrovascular hemodynamic correlates of aging in the Lou/c rat: a model of healthy aging. Neuroimage 56, 1892-1901. doi: 10.1016/j.neuroimage.2011.0 3.076

Everitt, B. J., and Robbins, T. W. (1997). Central cholinergic systems and cognition. Annu. Rev. Psychol. 48, 649-684. doi: 10.1146/annurev.psych.48.1.649

Fanselow, E. E., Richardson, K. A., and Connors, B. W. (2008). Selective, statedependent activation of somatostatin-expressing inhibitory interneurons in mouse neocortex. J. Neurophysiol. 100, 2640-2652. doi: 10.1152/jn.90691. 2008

Fu, Y., Kaneko, M., Tang, Y., Alvarez-Buylla, A., and Stryker, M. P. (2015). A cortical disinhibitory circuit for enhancing adult plasticity. elife 4:e05558. doi: 10.7554/eLife.05558

Furey, M. L., Pietrini, P., Haxby, J. V., and Drevets, W. C. (2008). Selective effects of cholinergic modulation on task performance during selective attention. Neuropsychopharmacology 33, 913-923. doi: 10.1038/sj.npp.1301461

Garait, B., Couturier, K., Servais, S., Letexier, D., Perrin, D., Batandier, C., et al. (2005). Fat intake reverses the beneficial effects of low caloric intake on skeletal muscle mitochondrial $\mathrm{H}(2) \mathrm{O}(2)$ production. Free Radic. Biol. Med. 39, 12491261. doi: 10.1016/j.freeradbiomed.2005.06.026

Gibson, G. E., Peterson, C., and Jenden, D. J. (1981). Brain acetylcholine synthesis declines with senescence. Science 213, 674-676. doi: 10.1126/science.7256270

Goldstone, R. L. (1998). Perceptual learning. Annu. Rev. Psychol. 49, 585-612.

Greene, C., Lee, H., and Thuret, S. (2019). In the long run: physical activity in early life and cognitive aging. Front. Neurosci. 13:884. doi: 10.3389/fnins.2019.0 0884

Greuel, J. M., Luhmann, H. J., and Singer, W. (1988). Pharmacological induction of use-dependent receptive field modifications in the visual cortex. Science 242, 74-77. doi: 10.1126/science.2902687

Gunn, R. N., Lammertsma, A. A., Hume, S. P., and Cunningham, V. J. (1997). Parametric imaging of ligand-receptor binding in PET using a simplified reference region model. Neuroimage 6, 279-287. doi: 10.1006/nimg.1997.0303
Hangya, B., Ranade, S. P., Lorenc, M., and Kepecs, A. (2015). Central cholinergic neurons are rapidly recruited by reinforcement feedback. Cell 162, 1155-1168. doi: 10.1016/j.cell.2015.07.057

Hasselmo, M. E., and Bower, J. M. (1993). Acetylcholine and memory. Trends Neurosci. 16, 218-222.

Hasselmo, M. E., and Sarter, M. (2011). Modes and models of forebrain cholinergic neuromodulation of cognition. Neuropsychopharmacology 36, 52-73. doi: 10. 1038/npp.2010.104

He, H.-Y., Hodos, W., and Quinlan, E. M. (2006). Visual deprivation reactivates rapid ocular dominance plasticity in adult visual cortex. J. Neurosci. 26, 29512955. doi: 10.1523/jneurosci.5554-05.2006

Kang, J. I., Groleau, M., Dotigny, F., Giguère, H., and Vaucher, E. (2014). Visual training paired with electrical stimulation of the basal forebrain improves orientation-selective visual acuity in the rat. Brain Struct. Funct. 219, 14931507. doi: 10.1007/s00429-013-0582-y

Kawaguchi, Y. (1997). Selective cholinergic modulation of cortical GABAergic cell subtypes. J. Neurophysiol. 78, 1743-1747. doi: 10.1152/jn.1997.78.3.1743

Kilbourn, M. R., Hockley, B., Lee, L., Sherman, P., Quesada, C., Frey, K. A., et al. (2009). Positron emission tomography imaging of (2R,3R)-5[(18)F]fluoroethoxybenzovesamicol in rat and monkey brain: a radioligand for the vesicular acetylcholine transporter. Nucl. Med. Biol. 36, 489-493. doi: 10.1016/j.nucmedbio.2009.02.007

Knudsen, E. I. (2004). Sensitive periods in the development of the brain and behavior. J. Cogn. Neurosci. 16, 1412-1425. doi: 10.1162/0898929042304796

Kollen, M., Stéphan, A., Faivre-Bauman, A., Loudes, C., Sinet, P.-M., Alliot, J., et al. (2010). Preserved memory capacities in aged Lou/C/Jall rats. Neurobiol. Aging 31, 129-142. doi: 10.1016/j.neurobiolaging.2008.03.010

Kuchibhotla, K. V., Gill, J. V., Lindsay, G. W., Papadoyannis, E. S., Field, R. E., Sten, T. A. H., et al. (2017). Parallel processing by cortical inhibition enables context-dependent behavior. Nat. Neurosci. 20, 62-71. doi: 10.1038/nn.4436

Liguz-Lecznar, M., Lehner, M., Kaliszewska, A., Zakrzewska, R., Sobolewska, A., and Kossut, M. (2015). Altered glutamate/GABA equilibrium in aged mice cortex influences cortical plasticity. Brain Struct. Funct. 220, 1681-1693. doi: 10.1007/s00429-014-0752-756

Liu, C.-H., Coleman, J. E., Davoudi, H., Zhang, K., and Hussain Shuler, M. G. (2015). Selective activation of a putative reinforcement signal conditions cued interval timing in primary visual cortex. Curr. Biol. 25, 1551-1561. doi: 10.1016/ j.cub.2015.04.028

McGeer, P. L., McGeer, E. G., Suzuki, J., Dolman, C. E., and Nagai, T. (1984). Aging. Alzheimer's disease, and the cholinergic system of the basal forebrain. Neurology $34,741-745$.

Mishra, J., de Villers-Sidani, E., Merzenich, M., and Gazzaley, A. (2014). Adaptive training diminishes distractibility in aging across species. Neuron 84, 10911103. doi: 10.1016/j.neuron.2014.10.034

Mulholland, G. K., Jung, Y.-W., Wieland, D. M., Kilbourn, M. R., and Kuhl, D. E. (1993). Synthesis of [18F]fluoroethoxy-benzovesamicol, a radiotracer for cholinergic neurons. J. Label Compd. Radiopharm. 33, 583-591. doi: 10.1002/ jlcr.2580330704

Mulholland, G. K., Wieland, D. M., Kilbourn, M. R., Frey, K. A., Sherman, P. S., Carey, J. E., et al. (1998). [18F]fluoroethoxy-benzovesamicol, a PET radiotracer for the vesicular acetylcholine transporter and cholinergic synapses. Synapse 30, 263-274. doi: 10.1002/(sici)1098-2396(199811)30:3<263::aid-syn4>3.0.co;2-9

Mzengeza, S., Massarweh, G., Rosa Neto, P., Soucy, J.-P., and Bedard, M. A. (2007). Radiosynthesis of [18F]FEOV and in vivo PET imaging of acetylcholine vesicular transporter in the rat. J. Cereb. Blood Flow Metab. 27, 10U-17U.

Nejad-Davarani, S., Koeppe, R. A., Albin, R. L., Frey, K. A., Müller, M. L. T. M., and Bohnen, N. I. (2019). Quantification of brain cholinergic denervation in dementia with lewy bodies using PET imaging with [F]-FEOBV. Mol. Psychiatry 24, 322-327. doi: 10.1038/s41380-018-0130-5

Obermayer, J., Verhoog, M. B., Luchicchi, A., and Mansvelder, H. D. (2017). Cholinergic modulation of cortical microcircuits is layer-specific: evidence from rodent. Monkey Hum. Brain. Front. Neural. Circuits 11:100. doi: 10.3389/fncir. 2017.00100

Ohl, F. W., and Scheich, H. (2005). Learning-induced plasticity in animal and human auditory cortex. Curr. Opin. Neurobiol. 15, 470-477. doi: 10.1016/j. conb.2005.07.002

Parent, M., Bedard, M.-A., Aliaga, A., Soucy, J.-P., Landry St-Pierre, E., Cyr, M., et al. (2012). PET imaging of cholinergic deficits in rats using 
[18F]fluoroethoxybenzovesamicol ([18F]FEOBV). Neuroimage 62, 555-561. doi: 10.1016/j.neuroimage.2012.04.032

Parent, M. J., Bedard, M.-A., Aliaga, A., Minuzzi, L., Mechawar, N., Soucy, J.P., et al. (2013a). Cholinergic depletion in Alzheimer's disease shown by [(18) F]FEOBV autoradiography. Int. J. Mol. Imag. 2013:205045.

Parent, M. J., Cyr, M., Aliaga, A., Kostikov, A., Schirrmacher, E., Soucy, J.-P., et al. (2013b). Concordance between in vivo and postmortem measurements of cholinergic denervation in rats using PET with [18F]FEOBV and choline acetyltransferase immunochemistry. EJNMMI Res. 3:70. doi: 10.1186/2191$219 \mathrm{x}-3-70$

Paxinos, G., and Watson, C. (2013). The Rat Brain in Stereotaxic Coordinates: Hard Cover Edition. Cambridge, MA: Academic Press.

Perry, E. K. (1980). The cholinergic system in old age and Alzheimer's disease. Age Ageing 9, 1-8. doi: 10.1093/ageing/9.1.1

Rokem, A., and Silver, M. A. (2010). Cholinergic enhancement augments magnitude and specificity of visual perceptual learning in healthy humans. Curr. Biol. 20, 1723-1728. doi: 10.1016/j.cub.2010.08.027

Rokem, A., and Silver, M. A. (2013). The benefits of cholinergic enhancement during perceptual learning are long-lasting. Front. Comput. Neurosci. 7:66. doi: 10.3389/fncom.2013.00066

Rubins, D. J., Melega, W. P., Lacan, G., Way, B., Plenevaux, A., Luxen, A., et al. (2003). Development and evaluation of an automated atlas-based image analysis method for microPET studies of the rat brain. Neuroimage 20, 21002118. doi: 10.1016/j.neuroimage.2003.07.011

Sarter, M., Hasselmo, M. E., Bruno, J. P., and Givens, B. (2005). Unraveling the attentional functions of cortical cholinergic inputs: interactions between signaldriven and cognitive modulation of signal detection. Brain Res. Brain Res. Rev. 48, 98-111. doi: 10.1016/j.brainresrev.2004.08.006

Schäfer, M. K., Weihe, E., Varoqui, H., Eiden, L. E., and Erickson, J. D. (1994). Distribution of the vesicular acetylcholine transporter (VAChT) in the central and peripheral nervous systems of the rat. J. Mol. Neurosci. 5, 1-26. doi: $10.1007 / \mathrm{bf02736691}$

Schliebs, R., and Arendt, T. (2011). The cholinergic system in aging and neuronal degeneration. Behav. Brain Res. 221, 555-563. doi: 10.1016/j.bbr.2010.11.058

Van Brussel, L., Gerits, A., and Arckens, L. (2011). Evidence for cross-modal plasticity in adult mouse visual cortex following monocular enucleation. Cereb. Cortex 21, 2133-2146. doi: 10.1093/cercor/bhq286

Verhoog, M. B., Obermayer, J., Kortleven, C. A., Wilbers, R., Wester, J., Baayen, J. C., et al. (2016). Layer-specific cholinergic control of human and mouse cortical synaptic plasticity. Nat. Commun. 7:12826. doi: 10.1038/ncomms12826

Voss, P., Thomas, M., Chou, Y. C., Cisneros-Franco, J. M., Ouellet, L., and de Villers-Sidani, E. (2016). Pairing cholinergic enhancement with perceptual training promotes recovery of age-related changes in rat primary auditory cortex. Neural Plast. 2016, 1801979. doi: 10.1155/2016/1801979

Zhou, X., Panizzutti, R., de Villers-Sidani, E., Madeira, C., and Merzenich, M. M. (2011). Natural restoration of critical period plasticity in the juvenile and adult primary auditory cortex. J. Neurosci. 31, 5625-5634. doi: 10.1523/JNEUROSCI. 6470-10.2011

Conflict of Interest: The authors declare that the research was conducted in the absence of any commercial or financial relationships that could be construed as a potential conflict of interest.

Copyright (๑) 2020 Cisneros-Franco, Voss, Kang, Thomas, Côté, Ross, Gaudreau, Rudko, Rosa-Neto and de-Villers-Sidani. This is an open-access article distributed under the terms of the Creative Commons Attribution License (CC BY). The use, distribution or reproduction in other forums is permitted, provided the original author(s) and the copyright owner(s) are credited and that the original publication in this journal is cited, in accordance with accepted academic practice. No use, distribution or reproduction is permitted which does not comply with these terms. 